\title{
IL-12 Enhances Immune Response by Modulation of Myeloid Derived Suppressor Cells in Tumor Microenvironment
}

\author{
Ji-Na Choi, Eun Gene Sun, and Sang-Hee Cho* \\ Department of Hematology-Oncology, Chonnam National University Hwasun Hospital, Hwasun, Korea
}

\begin{abstract}
Myeloid derived suppressor cells (MDSCs) are a heterogenous population of immature cells that play a critical role in tumor associated immune suppression. In tumor conditions, the population of MDSCs increases. The main feature of these cells is their ability to suppress the $\mathrm{T}$ cell response in antigen specific or nonspecific manners depending on the condition of T cell activation. IL-12 can modulate MDSC in preliminary reports, so we investigated how IL-12 can affect MDSC in a tumor microenvironment. After implanting tumor based cells on syngeneic host, 4T-1/BALB/c or EL4/C57BL6 mice, MDSCs (Gr1+CD11b+) were isolated from splenocytes. Isolated MDSCs were treated with GM-CSF with or without IL-12 and analyzed based on their phenotypes and functions. Treatment of MDSC with IL-12 increased co-stimulatory molecules of CD80, CD86, OX-40L, enhancing the DC phenotype (CD11c) and maturation markers such as $\mathrm{p}-\mathrm{NF}-\mathrm{kB}$ and $\mathrm{p}$-GSK3 3 . In addition to a change of surface markers, T-cell suppressive function of MDSC after IL-12 treatment was significantly improved compared with the control MDSC. In addition, PD-L1+F4/80+ macrophages, which show aninhibitory effect in phagocytosis, were decreased after IL-12 treatment. The changes of cell surface expression of CD80, CD86, MHC class II were also shown in vivo. Our results showed that the IL-12 can modulate MDSC into APC and recover the macrophage function. These results suggested that IL-12 plays a role in improving the tumor immune microenvironment through MDSC modulation.
\end{abstract}

Key Words: Myeloid-Derived Suppressor Cells; Interleukin-12; Tumor Microenvironment

This is an Open Access article distributed under the terms of the Creative Commons Attribution Non-Commercial License (http://creativecommons.org/licenses/by-nc/4.0) which permits unrestricted non-commercial use, distribution, and reproduction in any medium, provided the original work is properly cited.

\section{Article History:}

Received September 7, 2018

Revised October 2, 2018

Accepted October 15, 2018

\section{Corresponding Author:}

Sang-Hee Cho

Department of Hematology-Oncology, Chonnam National University Hwasun Hospital, 322 Seoyang-ro, Hwasun-eup, Hwasun 58128, Korea Tel: $+82-61-379-7633$

Fax: +82-61-379-7628

E-mail: shcho@jnu.ac.kr

\section{INTRODUCTION}

The fact that immune and tumor progression are closely linked is no longer a new paradigm. Based on the recent data about preclinical or clinical experiments for the cancer-immune network, the approachs to enhance the pro-inflammatory effect against tumor cells or suppress the tumor microenvironment (TME) could be important ways to find new directions for cancer treatment.

Myeloid derived suppressor cells (MDSC) are well known as the major parts of the TME composed of macrophages, granulocytes, and dendritic cells (DCs) that are in the early stages of cancer development. ${ }^{1}$ In physiologic conditions, they are present about $20-30 \%$ of bone marrow and more than $4 \%$ of nucleated cells in the spleen. ${ }^{2}$ However, under pathologic conditions such as infections, stress, and cancer, those cells accumulate at higher levels. The main characteristics of these cells are potent immune suppressive activity influenced during carcinogenesis and that these cells accumulate without differentiation to the functional mature cells. There are two major subsets of MDSC based on their phenotypes and morphological features: polymorphonuclear MDSC (PMN-MDSC) are similar to neutrophils, whereas monocytic MDSC (M-MDSC) are similar to monocytes. Although the proportions of subtypes of MDSC may be different depending on the type of cancer, their immunosuppressive role in tumor environments is similar.

MDSCs are generated from bone marrow derived cells (BMDC) and peripheral blood monocyte cells (PBMC) under multiple growth factors and cytokines. ${ }^{3}$ The recruitment of monocyte/M-MDSC or granulocyte/PMN-MDSC is 
regulated by chemokines produced by the tumors. Infiltrated MDSCs are accumulated and expanded by various cytokines/chemokines in tumor milieu without maturation. CCL2 and CXCL12 (SDF-1) usually recruit monocytes or M-MDSC to the tumor site and CXCL5, CXCL6, CXCL8 and CCL 15 recruit PMN-MDSC. ${ }^{4}$

The MDSCs produced by this process secrete several substances such as NO and ROS to create an immunocompromising tumor environment and elimination of key nutrition factors needed for $\mathrm{T}$ cell proliferation by depleting L-arginine, sequestering L-cystine, or reducing tryptophan levels due to the activity of indoleamine $2,3 \mathrm{di}$ oxygenase (IDO). ${ }^{5-7}$ MDSC also produces immunosuppressive cytokines such as IL-10 and TGF-beta, induces T regulatory cells (Tregs), affects Natural Killer (NK) cell function, and uses other suppressive mechanisms. ${ }^{5}$ In addition, splenic and tumoral infiltrating MDSC decreases the antigen-specific immune response of $\mathrm{T}$ cells in mice and patients with head and neck cancer. ${ }^{1}$

Thus, it appears that the modulation of MDSC tumor environment is another important method for tumor control. Based on this concept, we can hypothesize that the modulation of MDSC could be attenuated to the immune suppressive function in TME.

Among the various cytokines, the interleukin 12 (IL-12) is unique. With IL-23, IL-12 is a main proinflammatory and prostimulatory cytokine with key roles in the development of the Th1 and Th17 subsets of helper T cells, separately. ${ }^{8}$ IL-12 induces production of IFN- $\gamma$ by T cells primes additional antigen-presenting cells and facilitates Th1 differentiation. IL-12 can also induce the production of IFN- $\gamma$ by natural killer cells and a potent positive feedback loop to influence other immune cells. ${ }^{9}$ Intra-tumoral IL-12 can induce the apoptosis of regulatory $\mathrm{T}$ cells and impair memory CD8+ T cells permitting an influx of activated tumoricidal CD4+ and CD8+ T cells. ${ }^{10}$ In addition to T cells, macrophages are also regulated by IL-12. Macrophages are another important population in TME. Especially, tumor-associated macrophages (TAMs) correlate with a poor prognosis in human cancer. ${ }^{11}$ TAMs are often thought to polarize towards an inflammatory 'M1' or pro-tumor 'M2 state, depending on their environmental stimuli. Previously, IL-12 receptors (IL-12R $\beta 1$, IL-12R $\beta 2$ ) are expressed on surface of MDSCs and macrophages with a positive regulatory function in IL-12. ${ }^{12,13}$ These findings suggest that IL-12 may improve immune suppressive environments and promote immune stimulation under tumor conditions.

Therefore, this study was performed to explore how IL-12 can affect MDSC in tumor environments and the possibility to reverse the immunosuppressive function of MDSC by inducing immune stimulatory cells such as antigen presenting cells (APCs) or macrophages.

\section{MATERIALS AND METHODS}

\section{Reagents and compounds}

Penicillin-streptomycin, fetal bovine serum (FBS) and
RPMI 1640 were purchased from Gibco Co (Uxbridege, U.K). Phosphate buffer solution (PBS), red blood cell (RBC) lysing buffer and $\beta$-mercaptoethanol were obtained from Sigma (St.Louis, MO). Recombinant mouse GM-CSF and IL-12 were obtained from BD pharMingen (SanDiego, CA).

The antibodies used for flow cytometry: anti-CD86, anti-CD80, anti-OX40L, anti-Gr-1, anti-CD11b, anti-CD11c, anti-F4/80, anti-PD-L1, anti-MHC-class II, anti-INF- $\gamma$, and anti-CD16/CD32 mouse Fc blocker were obtained from BD pharMingen (SanDiego, CA). The antibodies used for Western blotting: anti-AKT, anti-phospho-AKT, an-

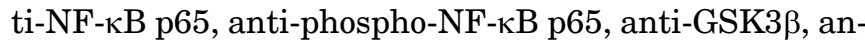
ti-phospho-GSK3 $\beta$, anti-phospho-STAT3, and anti- $\beta$-actin were purchase from Cell Signaling Technology (Danvers, MA, USA). Pan T cell isolation kit and myeloid-derived suppressor cell isolation kit were purchased from Miltenyi Biotec (Bergisch Gladbach, Germany). Mouse IL-10 and IL-6 ELISA kit were purchased from R\&D system (Minneapolis, MN). Anti-CD3, and anti-CD28 were obtained from BD pharMingen (SanDiego, CA).

\section{Cell, cell isolation and treatment}

The 4T1 murine mammary tumor cell line and EL4 murine lymphoma cell line were purchased from American Type Culture Collection (ATCC). The 4T1 and EL4 cell lines were cultured in RPMI 1640 medium, supplemented with $10 \%$ fetal bovine serum and $1 \%$ penicillin-streptomycin, at $37^{\circ} \mathrm{C}$ in $5 \% \mathrm{CO}^{2}$ atmosphere.

For MDSC and pan T-cell isolation, spleen tissues were removed from tumor bearing or naïve mice, respectively. Splenocytes were isolated by straining mashed spleen through a $70 \mu \mathrm{m}$ cell strainer into supplemented RPMI 1640 medium containing $50 \mu \mathrm{M} \beta$-mercaptoethanol. RBCs were then lysed with RBC lysis buffer. Subsequently splenocytes were resuspended in supplemented RPMI 1640 medium containing $50 \mu \mathrm{M} \beta$-mercaptoethanol and $70 \mu \mathrm{m}$ nylon cell strainer was used to obtain a uniform single-cell suspension.

MDSC isolation was conducted using total splenocytes from tumor-bearing mice and a Myeloid-derived suppressor cell isolation kit (MACS Mitenyl Biotec.), following the manufacturer's protocol. Gr-1 high Ly-6G+ cells were used for in vitro treatment experiment and T-cell suppression assay. The purity of the isolated MDSC was > 99\%. Pan T cells were isolated using total splenocytes from naïve mice and a Pan T cell isolation kit (MACS Mitenyl Biotec.), according to the manufacturer's protocol.

For the in vitro treatment experiment and flow cytometry analysis, MDSCs from tumor-bearing mice were cultured in the absence or presence of recombinant mouse IL-12 (10 ng/mL) for indicated time.

\section{Animal model}

All animal procedures were approved by The Chonnam National University Medical School Research Institutional Animal Care and Use Committee. 6-8-week-old BALB/c female mice and C57BL/6 mice were purchased from Orient 
(Seong-nam, Korea) and maintained in a standard cabinet under specific pathogen-free conditions.

4T1 and EL4 tumor-bearing mouse models were used for MDSC generation and the in vivo study of Adenovirus encoding mouse IL-12 (Ad mIL-12). 4T1 murine mammary tumor cells $\left(10^{6}\right.$ cells/fat pad) were implanted subcutaneously into the mammary fat pads of BALB/c mice. In brief, a syringe with a $26 \mathrm{G}$ needle was used to inject the cell suspension directly into the mammary gland. Inoculations were conducted within $30 \mathrm{~min}$ of preparation of cell suspensions. Tumor volume was measured with a caliper every other day, and determined based on the equation $\pi / 6$ $\left(a \times b^{2}\right)$, where $a$ is the largest tumor diameter and $\mathrm{b}$ is the smallest. When the volume of the primary tumors reached $1000 \mathrm{~mm}^{3}$, the $4 \mathrm{~T} 1$ tumor bearing mice were sacrificed following anesthetization and spleen tissues were obtained for MDSC isolation.

For the in vivo study of Ad mIL-12, EL4 murine lymphoma cells $\left(10^{6}\right.$ cells $/ 100 \mu \mathrm{L}$ PBS $)$ were injected subcutaneously into the right flank of C57BL/6 mice. When the average tumor volume reached $700 \mathrm{~mm}^{3}$, mice were randomly divided into two groups receiving Ad-vector or Ad-mIL-12. Adenovirus encoding mIL-12 ( $10^{10}$ plaque-forming units) was injected intravenously in EL4 tumor-bearing mice. After 1 day, spleen tissues were removed for splenocyte isolation and flow cytometry analysis.

\section{T-cell suppression assay (in vitro MDSC suppression assay)}

The suppressive capacity of MDSC was determined by co-culture with pan $\mathrm{T}$ cells. Isolated pan $\mathrm{T}$ cells from healthy mice and MDSCs from 4T1 tumor bearing mice were used as responder cells and stimulator cells, respectively. Responder and stimulator cells were then mixed at a 1:10 ratio and $T$ cell proliferation was assessed by thymidine incorporation. Briefly, $10^{6}$ splenic MDSCs in complete RPMI 1640 media were plated with $10^{5}$ pan T cells in a 96-well plate. Pan T cells were activated with anti-CD3 $(0.5 \mu \mathrm{g} / \mathrm{mL})$ and anti-CD28 $(0.5 \mu \mathrm{g} / \mathrm{mL})$ for 4 days and maintained with or without mouse IL-12 (10 ng/mL, R\&D systems) as previously reported. ${ }^{13}$ Subsequently, $\left[{ }^{3} \mathrm{H}\right]$ thymidine $(1 \mu \mathrm{Ci} /$ well $)$ was added to the wells for the last $16 \mathrm{hr}$ of the 4 day culture periods. Responses are expressed as the mean counts per minute (cpm).

\section{Flow cytometry}

Single-cell suspensions were prepared by sieving and gentle pipetting. After FACS buffer washing, cells were pre-incubated with anti-CD16/CD32 mouse Fc blocker and stained for $30 \mathrm{~min}$ at $4^{\circ} \mathrm{C}$ with fluorochrome-conjugated antibodies. Cells were washed thoroughly with FACS buffer between each step. Samples were collected with FACScalibur (BD pharMingen).

\section{Western blot analysis}

Protein levels were assessed by Western blotting. The to-
A
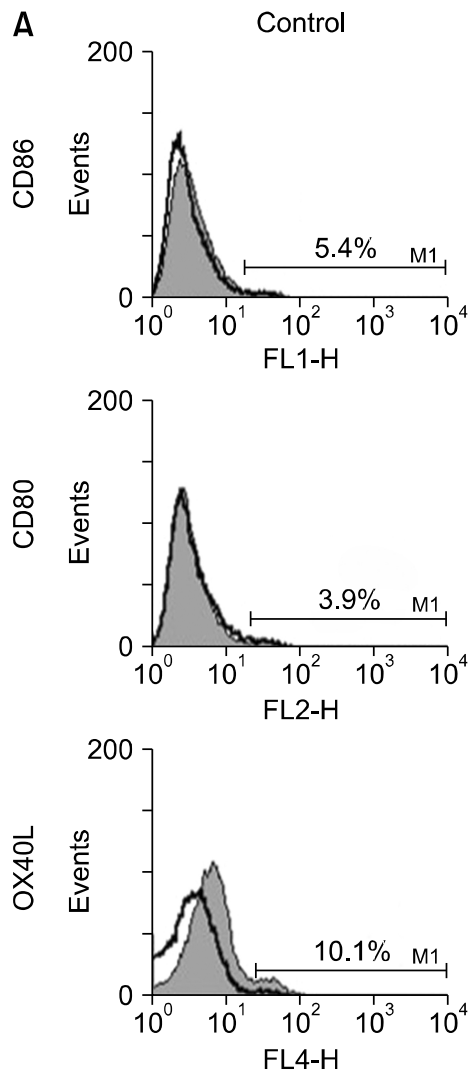

IL-12
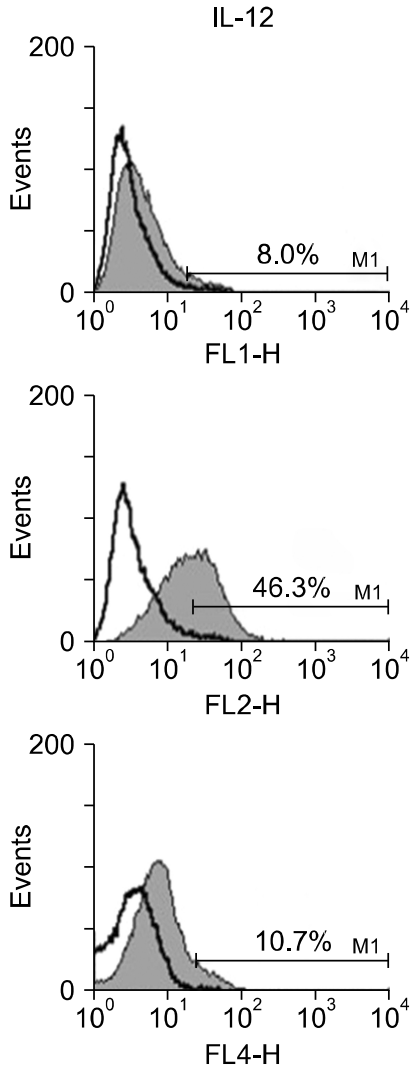

B

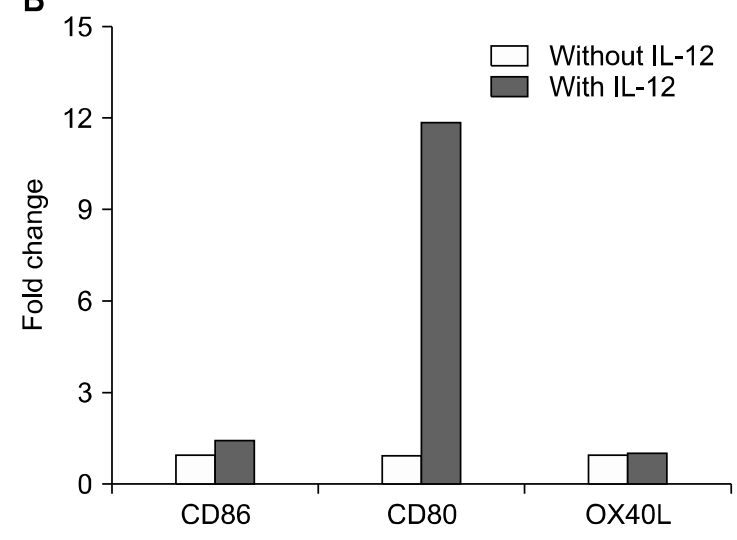

FiG. 1. Surface phenotypes of splenic MDSCs from tumor bearing mouse with IL-12 treatment. (A) Co-stimulatory molecules of spleen-derive MDSCs from 4T1/BALB/c tumor mouse model. Splenic MDSCs were cultured with IL-12 $(10 \mathrm{ng} / \mathrm{mL})$ and GM-CSF $(10 \mathrm{ng} / \mathrm{mL})$ for 1 day. (B) Fold changes of co-stimulatory molecules phenotype. 
tal proteins were extracted using M-PER mammalian protein extraction reagent containing protease and phosphatase inhibitors (Thermo Fisher Scientific, Rockford, IL, USA). Protein concentrations were determined using the BCA protein assay kit (Pierce, Rockford, IL, USA). Equal amounts of proteins were separated by SDS-PAGE and subsequently transferred from gels onto a polyvinylidene difluoride (PVDF) membrane (GE Healthcare Bio-sciences, Piscataway, NJ, USA), then immunoblotted with antibodies. Bound primary antibodies were visualized using horseradish peroxidase-conjugated second antibodies (Abcam, Cambridge, MA) with chemiluminescent substrate (Santa Cruz Biotechnology, CA, USA). Quantification of the immunoblotted bands was performed using Multi-gage software.

\section{ELISA}

After recombinant mouse IL-12 treatment, culture supernatants of MDSCs were collected for measurement of cytokine levels. Cytokine levels of IL-10 and IL-6 were measured using ELISA kits (BD science, San Jose, California, USA), according to the manufacturer's protocol. The optical density was read by a Microplate autoreader (Bio-Tek, Burlington, VT).

\section{Statistical analysis}

Statistical analysis was performed using the SPSS 12.0 software package (SPSS, Inc., Chicago, IL, USA). Statistical significance was assessed by Student's t-test. p-values less than 0.05 were considered statistically significant.

\section{RESULTS}

\section{Surface phenotypes of splenic MDSC can be modulated by IL-12 treatment}

To investigate whether MDSC could alter the phenotype of surface antigen under IL-12 treatment, spleen derived MDSCs from 4T1-tumor-bearing BALB/c mice were cultured with IL-12 (10 ng/mL) and GM-CSF (10 ng/mL) for 1 day and then checked for the expression of CD86, CD80 and OX40L (Fig. 1). Compared to CD86 and OX 40L, which were slightly increased after IL-12 treatment, they strongly expressed CD80. It suggested that IL-12 could induce the expression of surface co-stimulatory molecules in MDSC from tumor bearing mice.

\section{2. $\mathrm{IL-12}$ can attenuate $\mathrm{T}$ cell suppressive function of MDSC}

We next assessed the effect of CD8+ T cell stimulation on the increase of co-stimulatory molecules. As a negative control, MDSCs from tumor-bearing mouse splenocytes showed the inhibition of $\mathrm{T}$ cell proliferation as expected. However, MDSCs with IL-12 treatment reduced their suppressive activity on T cell stimulation (Fig. 2). Taken together, our data demonstrated that IL-12 induces the acquisition of antigen recognition function in MDSC and it can reverse the suppressive function of $\mathrm{T}$ cell stimulation.

\section{IL-12 mediated increase and maturation of dendritic cell from MDSC}

MDSCs are composed of immature myeloid cells such as monocyte, neutrophil and macrophages, as previously mentioned. Therefore, we hypothesized if MDSC from tumor bearing mice can reduce their immune suppressive function after IL-12 treatment and induce co-stimulatory molecules, the proportion of APCs such as DCs, may be increased. With this in mind, we next examined the change of CD11c population in splenocytes of normal and tumor bearing mice according to the $1 \mathrm{~L}-12$ treatment. Considering the comprehensive cellular interaction between immature myeloid cells, the proportion of CD11c was checked in IL-12 treated whole splenocytes of normal and tumor bearing mice, not in MDSC alone in normal and tumor bearing mice after IL-12 treatment. Although the difference was more pronounced in normal splenocytes than in tumoral splenocytes, the CD11c fraction was found to increase after IL-12 treatment in both normal and tumoral splenocytes (Fig. 3A, 3B). Next, we analyzed the DC differentiation markers to determine whether changes in cell surface antigens are associated with MDSC differentiation into APCs according to IL-12 treatment. In general, the expressions of $\mathrm{p}-\mathrm{NF}-\mathrm{\kappa B}$ and p-Akt increase with DC maturation process. GSK3 $\beta$ inhibits DC maturation and p-GSK3 $\beta$ means the inactivation form of GSK3 $\beta .{ }^{14}$ Since DC are mainly differentiated from the M-MDSC subset in the MDSC population, it was necessary to identify the decrease of Ly6C as an M-MDSC marker addition to the increase of CD11c, and molecular signal changes related to DC maturation. Thus, splenic MDSCs from tumor bearing mice were cultured with IL-12 and checked Ly 6C expression. As results, the fraction of Ly6C in CD11b positive population decreased (Fig. 3C), and the expression of p-NF-kB and p-GSK3 $\beta$ increased in cultured MDSCs with IL-12 treatment (Fig. 3D). These results suggested that IL-12 can increase CD11c positive cells in sple-

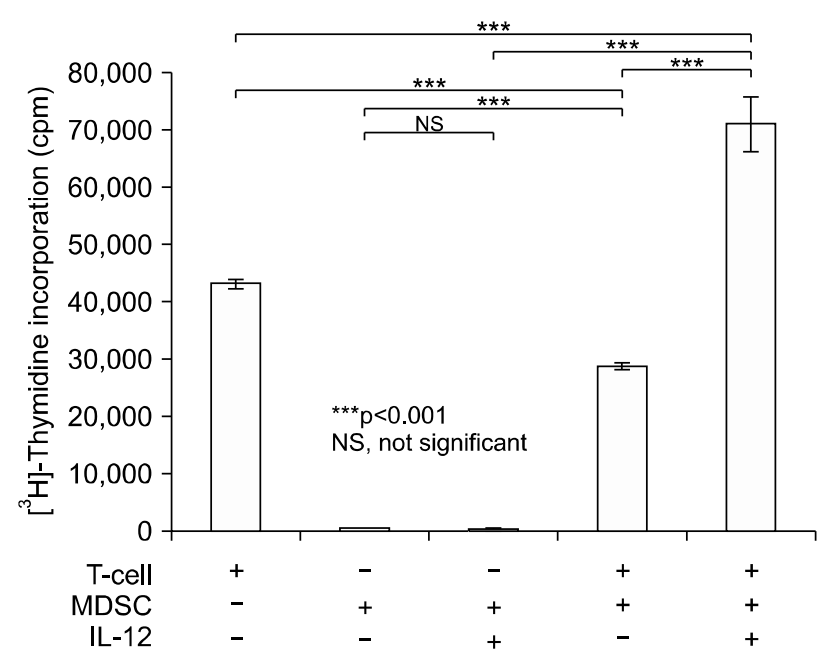

Fig. 2. IL-12 treated MDSCs stimulate CD8+ T cell proliferation. 

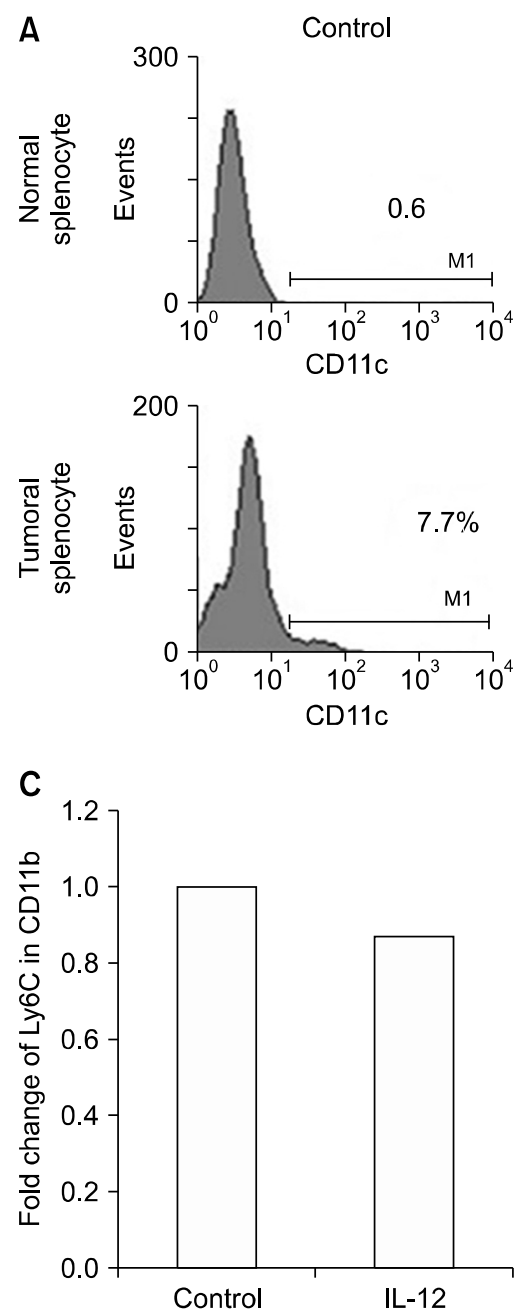
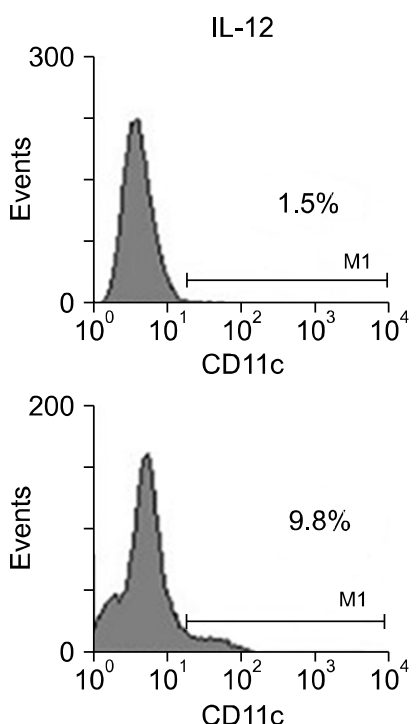

D

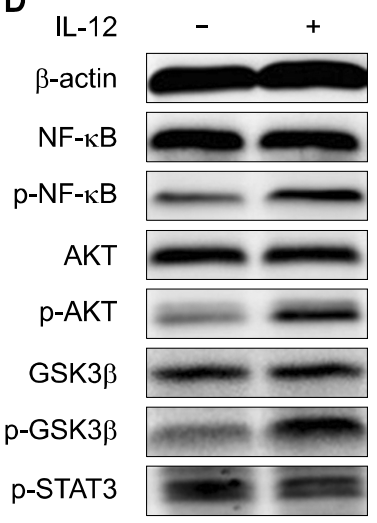

B

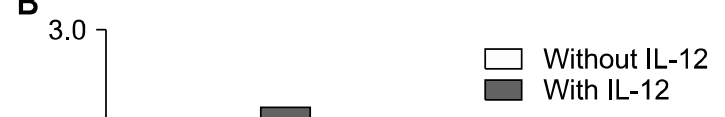
With IL-12

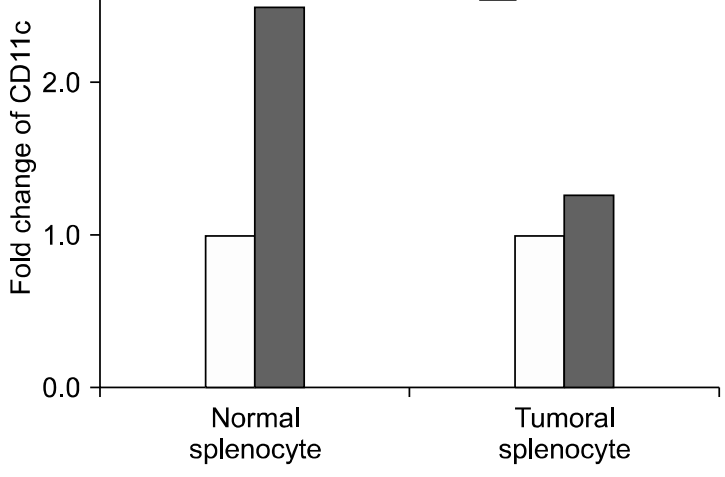

FiG. 3. Modulation of MDSCs into dendritic cells after IL-12 treatment. (A) CD11c fraction in tumor bearing splenocyte was increased after IL-12 treatment. (B) Fold changes of CD11c fraction in normal and tumoral splenocytes. (C) IL-12 decrease Ly6C expression in CD11b+MDSC. (D) Dendritic cell maturation markers are increased in splenic MDSC from tumor bearing mouse after IL-12 treatment. nocytes, and differentiation of MDSCs into DCs.

\section{IL-12 can effect on the differentiation of macrophage}

Macrophages are usually located aroud tumor sites, however, circulating macrophages are also located in spleen. IL-12 is secreated from activated macrophages, thus we investigated possible direct effects of IL-12 on macrophages differentation. In cytokine analysis associated with macrophage activity, IL-10, which inhibits macrophage activation, significantly decreased, but IL-6, which can influence the balance between M1 and M2, showed no significant change according to the IL-12 treatment (Fig. 4A). For further investigation, we analyzed the M2 surface antigen by FACS analysis. F4/80 is a major macrophagel marker and PD-L1 and F4/80 double positive macrophage can be regraded as M2 or tumor associated macrophage. ${ }^{15}$ In our study, the fraction of PD-L1 and F4/80 double positive cells decreased after IL-12 treatment in MDSCs from tumor bearing splenocyte (Fig. 4B). It means that M2 or tumor associated macrophage can be decreased after IL-12, and IL-10 secreted from macrophages such as M2 was also reduced.

\section{The expression of cell surface markers after $\mathrm{IL}-12$ treat- ment in vivo study}

Based on these results, we expanded the in vivo study to confirm our results. Similar to the in vitro study, CD80, CD86 and MHC class II were highly expressed in Ad mIL-12 treated MDSCs (more prominent of CD86 expression and MHC class II than CD80 expression). Interestingly, in the Ad mIL-12 treated MDSC, PD-L1 was significantly increased with the modest increase of INF- $\gamma$ compared to the control group (Fig. 5).

\section{DISCUSSION}

It is important to directly attack the tumor itself to reduce or inhibit tumor progression, but it is also important to strengthen the immune system. In particular, since the resistance mechanisms of targeted agents for tumors have been reported, the regulation of the tumor microenvironment (TME) is becoming more of an issue than ever before.

Among TME, myeloid cells, which are a highly diverse population, have a main role to serve as a key master in TME. Especially, the accumulation of MDSCs, which are important contributors to tumor progression and immune 
A

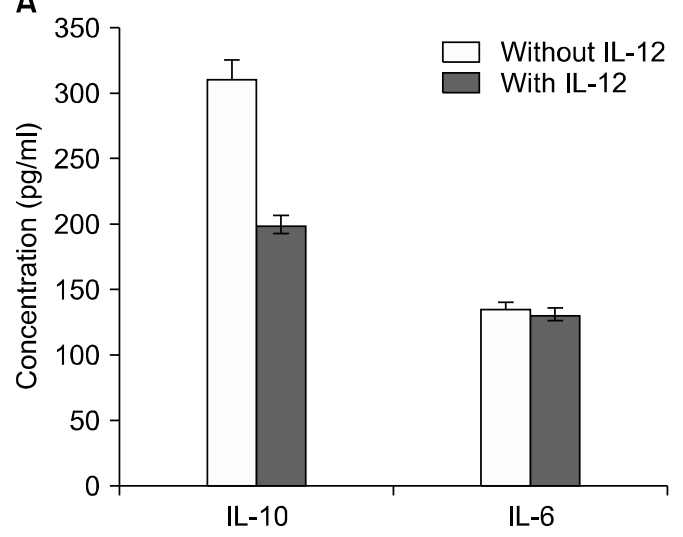

B

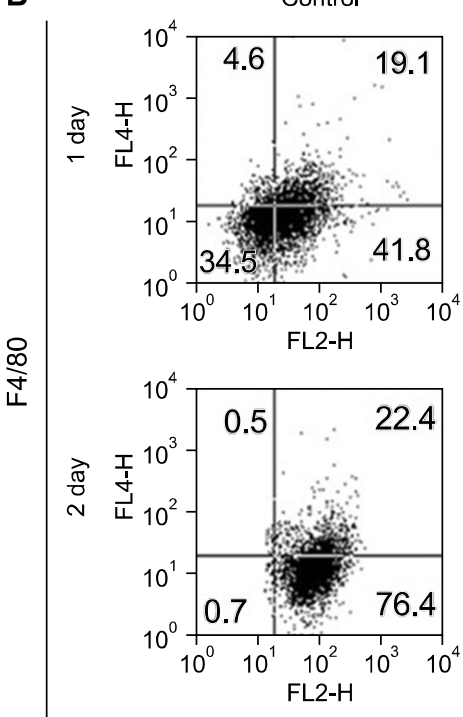

IL-12
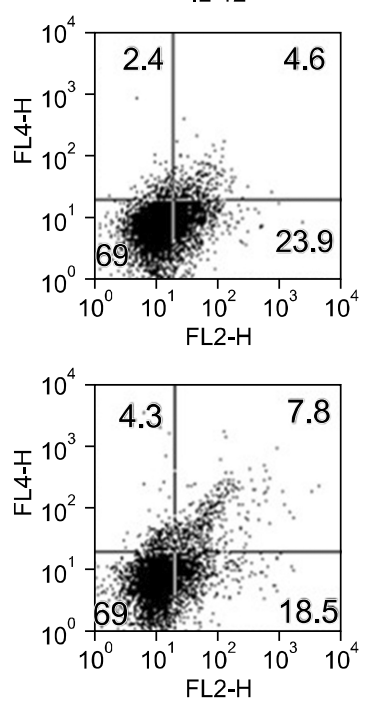

PD-L1

FiG. 4. Effect of IL-12 on the differentiation of macrophage. (A) Cytokine analysis in MDSC after IL-12 treatment. (B) Phenotypic changes of macrophage after IL-12 treatment.

suppression in cancer, is a big hurdle to restore TME. Until now, the mechanism of MDSC on TME or cancer cells have been extensively studied, and decreasing MDSC can induce tumor reduction. For example, Cytotoxic agents can induce apoptosis of MDSC and all-trans retinoic acid (ATRA) induce differentiation of MDSC into macrophages and/or DCs in vitro. ${ }^{16-19}$ Considering the large burden of MDSC in tumors, it would be a good way to induce not only the destruction of MDSC but also differentiation of MDSC into normal or immune stimulating cells to reverse TME.

IL-12 has the central role of immunoregulation in many ways of proinflammatory or prostimulatory cytokines. ${ }^{9,20}$ The antitumor effectors of IL-12 are thought to involve antiangiogenesis and increased production of IFN- $\gamma$ as well as the enhanced lytic abilities of CD8+ T cells, NK cells, and NKT cells to kill tumor cells. Not only promoting of generation of proinflammatory Thelper type 1 (TH1), Kerkar $\mathrm{SP}$ et al showed that adoptively transferring tumor antigen-specific CD8+ T cells engineered to secrete IL-12 can functionally remodel the TME into an activated inflammatory state.$^{21}$ In this report, IL-12 secreted from engineered CD8+ $\mathrm{T}$ cell could alter the intrinsic capabilities of professional APC residing within the tumor stroma including those of MDSC. Similar to our study, they showed the restoration of $\mathrm{T}$ cell function and the acquisition of antigen recognition function of MDSC under IL-12 secretion. We found the possibility of dynamic processes from MDSC into APC such as DCs. CD 80 and CD86 are expressed on antigen presenting cells and these are capable of binding both CD28 to stimulate T cells. In addition, DCs present antigen peptides in association with MHC II to naïve CCD4+ T lymphocytes. Our result showed that the expression of CD80, CD86 and MHC class II, increased after IL-12 treatment in MDSC both the in vitro and in vivo study. During DC maturation, Akt and NF- $\mathrm{KB}$ allow the increased expression of MHC II and co-stimulatory molecules, release of proinflammatory cytokines and chemokines, and DC migration and recruitment. This process leads to sustained $\mathrm{T}$ cell stimulation and IL-12 production. ${ }^{22,23}$ Our results also showed that IL-12 treated MDSC induce GSK- $3 \beta$ inhibition, this circuit of IL- 12 on MDSC modulation can affect the TME by a positive immune stimulatory function. This is a new finding that MDSC can differentiate into DCs, suggesting that MDSC can be converted into other cells.

Interestingly, PD-L1 expression increased after AdIL-12 treatment in our in vivo study and it is possibly related with increased INF- $\gamma$ following IL-12. ${ }^{24}$ Previous reports have demonstrated that IL-12 administration induced tumor regression in association with INF- $\gamma$ affects CD4 and CD8 T cell function. ${ }^{25}$ Taken together, INF- $\gamma$ plays an important role in the antitumor effect of IL-12. However, the increase of PD-L1 in MDSC after IL-12 treatment may be contradictory to the antitumor effect of IL-12 given that PD-L1 is immunosuppressive phenotype. In this regard, it is necessary to control the PD-L1 expression to sustain the improvement of the immune microenvironment after IL-12 treatment. Recently, it has been reported that PD-L1 expression in APCs, outside tumor tissues, plays an essential role in PD-1/PD-L1 blockade therapy. ${ }^{26}$ This is a new insight of PD-1/PD-L1 blockade therapy to target the host environment. Based on this evidence, our data suggests that IL-12 induces MDSC into APC and increases PD-L1 expression to improve response to PD-1/PD-L1 blockade therapy through changing a "cold" TME to a "hot" TME.

IL-12 also contributes to regulate macrophage function. M-MDSC can differentiate monocytes, DCs or macrophages. M-MDSC produces IL-10 and it represses macrophage 

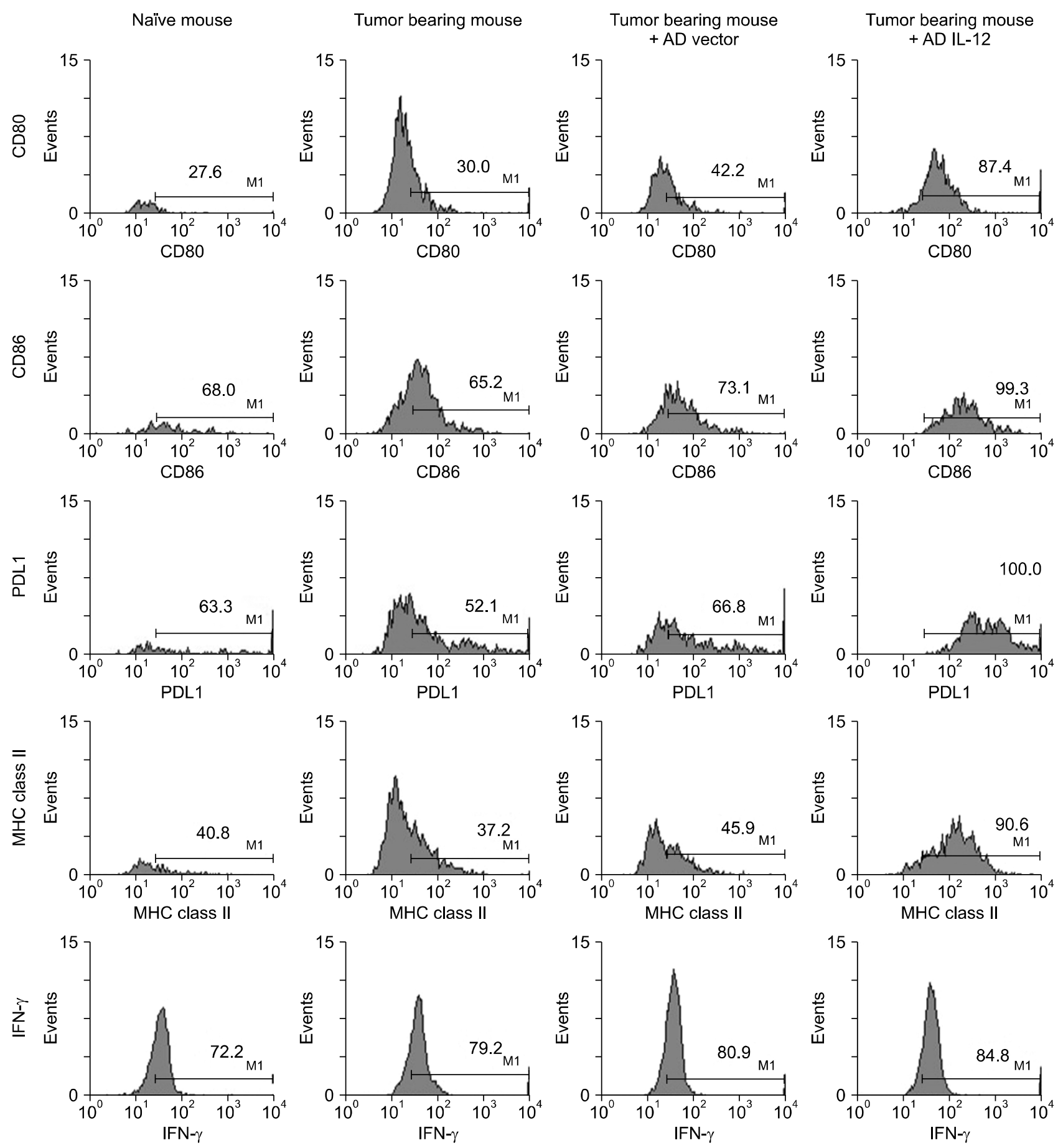

FiG. 5. In vivo characteristics of surface antigen molecules after adenoviral IL-12 injection.

function by decreasing MHC II expression and IL-12 excretion from macrophage. ${ }^{11}$ 'M1' macrophages, 'classically activated' macrophages which are activated by IFN- $\gamma$, express high levels of IL-12 and low levels of IL-10. By contrast, 'M2' macrophages, 'alternatively activated' macrophage which are activated by IL-4, IL-10, IL-13, express high levels of IL-10 and low levels of IL-12 and facilitate tumor progression. In our study, the level of IL-10 in supernatants of MDSC treated with IL-12 was higher than in that of the control group. It suggests that IL-12 can modu- late or differentiate MDSC into APC or macrophages, and those cells reduce the production of IL-10. In addition, the polarization of tumor associated macrophage (TAM) affects IL-12. TAM are often thought to polarize into an inflammatory "M1" or protumor "M2" states depending on TME status. Previous reports demonstrate that both mice and human TAMs express high level of PD-1, and that PD-1 expression on TAMs correlates with decreased phagocytosis, but PD-L1 removal increases PD-1+ TAM phagocytosis in vivo. ${ }^{15}$ In our study, among TAM (F4/80), 
PD-L1+ macrophages decreased after IL-12 treatment, it suggested that TAM function may be restored under IL-12 treatment.

Our report provides the possibility of improving TME with IL-12, but there are several limitations. First, despite changes in the surface antigen, no significant changes in MDSC proportions were observed following IL-12 treatment. One of causes for the phenomenon is thought to be the insufficient time for MDSC to be fully differentiated to APC after Ad mIL-12 injection to harvest samples. Secondly, the role of IL-12 in each of M-MDSC versus PMN-MDSC, or splenic MDSC versus tumoral MDSC, is required to discover the delicate interaction of DC differentiation after IL-12 treatment in further studies.

In conclusion, our results showed that IL-12 can modulate MDSC into APC and affect the macrophage phenotype when treated with IL-12 in TME. These results showed the possibility to attenuate immumosuppressive function of MDSCs and improve the immune surveillance in TME.

\section{ACKNOWLEDGEMENTS}

This research was supported by Research Institute of Medical Science, Chonnam National University.

\section{CONFLICT OF INTEREST STATEMENT}

None declared.

\section{REFERENCES}

1. Gabrilovich DI, Nagaraj S. Myeloid-derived suppressor cells as regulators of the immune system. Nat Rev Immunol 2009;9: 162-74.

2. Youn JI, Nagaraj S, Collazo M, Gabrilovich DI. Subsets of myeloid-derived suppressor cells in tumor-bearing mice. J Immunol 2008;181:5791-802.

3. Lechner MG, Liebertz DJ, Epstein AL. Characterization of cytokine-induced myeloid-derived suppressor cells from normal human peripheral blood mononuclear cells. J Immunol 2010;185: 2273-84.

4. Kumar V, Patel S, Tcyganov E, Gabrilovich DI. The nature of myeloid-derived suppressor cells in the tumor microenvironment. Trends Immunol 2016;37:208-20.

5. Gabrilovich DI, Ostrand-Rosenberg S, Bronte V. Coordinated regulation of myeloid cells by tumours. Nat Rev Immunol 2012;12: 253-68.

6. Bronte V, Zanovello P. Regulation of immune responses by L-arginine metabolism. Nat Rev Immunol 2005;5:641-54.

7. Nagaraj S, Gupta K, Pisarev V, Kinarsky L, Sherman S, Kang L, et al. Altered recognition of antigen is a mechanism of CD8+ T cell tolerance in cancer. Nat Med 2007;13:828-35.

8. Vignali DA, Kuchroo VK. IL-12 family cytokines: immunological playmakers. Nat Immunol 2012;13:722-8.

9. Takemoto N, Intlekofer AM, Northrup JT, Wherry EJ, Reiner SL. Cutting edge: IL-12 inversely regulates T-bet and eomesodermin expression during pathogen-induced CD8+ T cell differentiation. J Immunol 2006;177:7515-9.

10. Mazzolini G, Alfaro C, Sangro B, Feijoó E, Ruiz J, Benito A, et al. Intratumoral injection of dendritic cells engineered to secrete interleukin-12 by recombinant adenovirus in patients with metastatic gastrointestinal carcinomas. J Clin Oncol 2005;23:9991010.

11. Sica A, Schioppa T, Mantovani A, Allavena P. Tumour-associated macrophages are a distinct M2 polarised population promoting tumour progression: potential targets of anti-cancer therapy. Eur J Cancer 2006;42:717-27.

12. Ma X, Yan W, Zheng H, Du Q, Zhang L, Ban Y, et al. Regulation of IL-10 and IL-12 production and function in macrophages and dendritic cells. F1000Res 2015;4:1465.

13. Steding CE, Wu ST, Zhang Y, Jeng MH, Elzey BD, Kao C. The role of interleukin-12 on modulating myeloid-derived suppressor cells, increasing overall survival and reducing metastasis. Immunology 2011;133:221-38.

14. Alessandrini A, De Haseth S, Fray M, Miyajima M, Colvin RB, Williams WW, et al. Dendritic cell maturation occurs through the inhibition of GSK-3 3 . Cell Immunol 2011;270:114-25.

15. Gordon SR, Maute RL, Dulken BW, Hutter G, George BM, McCracken MN, et al. PD-1 expression by tumour-associated macrophages inhibits phagocytosis and tumour immunity. Nature 2017;545:495-9.

16. Le HK, Graham L, Cha E, Morales JK, Manjili MH, Bear HD. Gemcitabine directly inhibits myeloid derived suppressor cells in $\mathrm{BALB} / \mathrm{c}$ mice bearing $4 \mathrm{~T} 1$ mammary carcinoma and augments expansion of T cells from tumor-bearing mice. Int Immunopharmacol 2009;9:900-9.

17. Vincent J, Mignot G, Chalmin F, Ladoire S, Bruchard M, Chevriaux A, et al. 5-Fluorouracil selectively kills tumor-associated myeloid-derived suppressor cells resulting in enhanced $\mathrm{T}$ cell-dependent antitumor immunity. Cancer Res 2010;70:3052-61.

18. Ko JS, Zea AH, Rini BI, Ireland JL, Elson P, Cohen P, et al. Sunitinib mediates reversal of myeloid-derived suppressor cell accumulation in renal cell carcinoma patients. Clin Cancer Res 2009;15:2148-57.

19. Bauer R, Udonta F, Wroblewski M, Ben-Batalla I, Santos IM, Taverna F, et al. Blockade of myeloid-derived suppressor cell expansion with all-trans retinoic acid increases the efficacy of antiangiogenic Therapy. Cancer Res 2018;78:3220-32.

20. Smyth MJ, Taniguchi M, Street SE. The anti-tumor activity of IL-12: mechanisms of innate immunity that are model and dose dependent. J Immunol 2000;165:2665-70.

21. Kerkar SP, Goldszmid RS, Muranski P, Chinnasamy D, Yu Z, Reger RN, et al. IL-12 triggers a programmatic change in dysfunctional myeloid-derived cells within mouse tumors. J Clin Invest 2011;121:4746-57.

22. Bottero V, Withoff S, Verma IM. NF-kappaB and the regulation of hematopoiesis. Cell Death Differ 2006;13:785-97.

23. Zhang TY, Daynes RA. Glucocorticoid conditioning of myeloid progenitors enhances TLR4 signaling via negative regulation of the phosphatidylinositol 3-kinase-Akt pathway. J Immunol 2007;178:2517-26.

24. Lee SJ, Jang BC, Lee SW, Yang YI, Suh SI, Park YM, et al. 
Interferon regulatory factor-1 is prerequisite to the constitutive expression and IFN-gamma-induced upregulation of B7-H1 (CD274). FEBS Lett 2006;580:755-62.

25. Nastala CL, Edington HD, McKinney TG, Tahara H, Nalensnik MA, Brunda MJ, et al. Recombinant IL-12 administration in- duces tumor regression in association with IFN-gamma production. J Immunol 1994;153:1697-706.

26. Tang H, Liang Y, Anders RA, Taube JM, Qiu X, Mulgaonkar A, et al. PD-L1 on host cells is essential for PD-L1 blockade-mediated tumor regression. J Clin Invest 2018;128:580-8. 